\title{
Lateral Pharyngeal Diverticulum presenting with Dysphagia
}

N Berhanu, K Philipos, T Ayalew

Cardiothoracic Surgery Unit, Department of Surgery, Addis Ababa University, Addis Ababa, Ethiopia

Correspondence to: Dr. Philipos Kidane, Email: <philiposkidane@gmail.com>

Lateral pharyngeal diverticulum (Pharyngocele) is the protrusion of pharyngeal mucosa through the pharyngeal wall, usually through either of two weak areas in the pharyngeal wall as an acquired or congenital case. Lateral diverticula are very rare and not to be mistaken for the rather more frequent and abundantly reported cases of posterior pharyngo-esophageal diverticula (Zenker's). Here, we present a case of this very rare condition in a young boy who presented with severe dysphagia since childhood.

Key words: Lateral, pharyngeal, diverticulum dysphagia

DOI: http://dx.doi.org/10.4314/ecajs.v21i3.19

\section{Case Summary}

A 16 year old male patient presented to our hospital with difficulty of swallowing of solid and liquid diet since childhood with worsening of these symptoms since the past three years. Patient swallows with significant effort while noticing bulging of his neck on the right side. There was no history of cardiac, respiratory or neurologic condition in the past, no history of trauma, surgery or neck infection

Physical findings at admission included stable vital signs, otherwise unremarkable. Pertinent finding was noticed upon attempt at swallowing and with Valsalva maneuver with significant bulging of the right lateral neck which is cystic and reducible (Figure 1). No neck swelling on the left side.

Hence with the diagnosis of dysphagia secondary to lateral pharyngeal diverticulum he was investigated. Routine lab work was normal. Barium swallow revealed an out-pouching on the right lateral side of the hypopharynx on the right pyriform recess (Figure 2). CT scan of the neck also revealed the diverticulum. On Endoscopy, the right side recess looked distorted with bulging mucosal folds and it was not possible to intubate the esophagus.

The patient was subsequently operated with an initial incision made along the right anterior border of the sternocleidomastoid muscle and dissection was made to the level of the diverticulum which was arising between the middle and inferior pharyngeal constrictor muscles and the thyro-hyoid membrane. The diverticulum was subsequently opened and attempt was made to pass in a nasogastric tube and failed due to a tightly constricted cricopharyngeus muscle (Figure 3). Skin incision was then extended along the anterior border of the left sternocleidomastoid muscle and and dissection made to the level of the cervical esophagus which was mobilized and the right lobe of the thyroid gland was removed to gain better exposure of the cricopharyngeal junction.
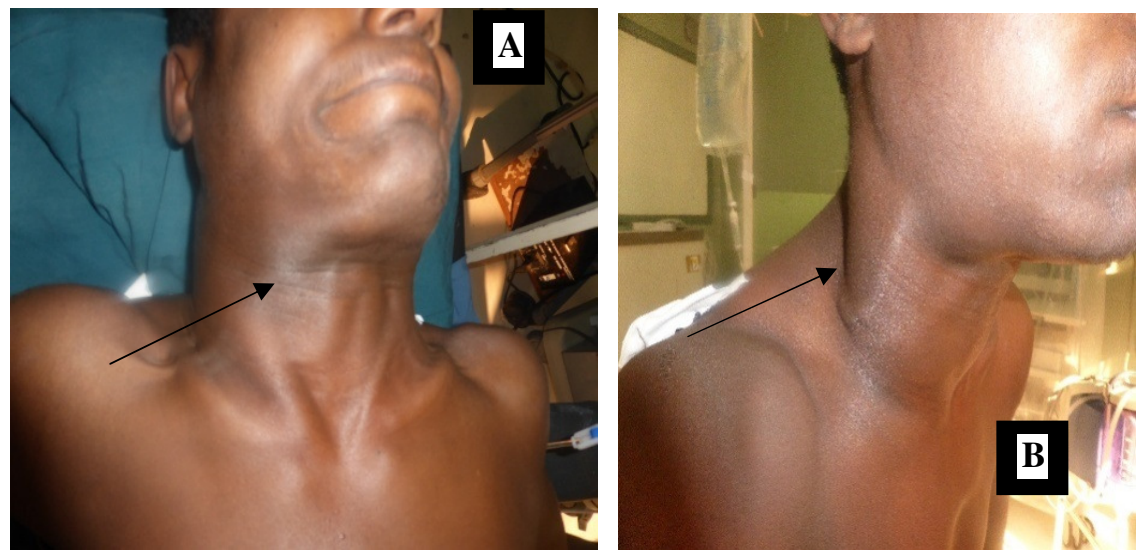

Fig 1. A and B Bulging right lateral neck mass during Valsalva maneuver (Arrows) 
Myotomy was done on the cricopharyngeus muscle and the diverticulum excised and closed in two layers after nasogastric tube was inserted. Cervical fascia and platysma was closed in layers after a glove drain was placed deep in the wound. After the patient was extubated and taken to the post anesthesia recovery room he developed respiratory arrest and was reintubated again with about 1.5 litres of hemorrhagic fluid sucked out through the endotracheal tube. Hence with the diagnosis of aspiration with pulmonary edema and possibly transient recurrent nerve palsy he was kept in the ICU where he was extubated on his $4^{\text {th }}$ post-operative day and recovered well to be discharged. He was evaluated on subsequent visits and is able to swallow both solids and liquids.
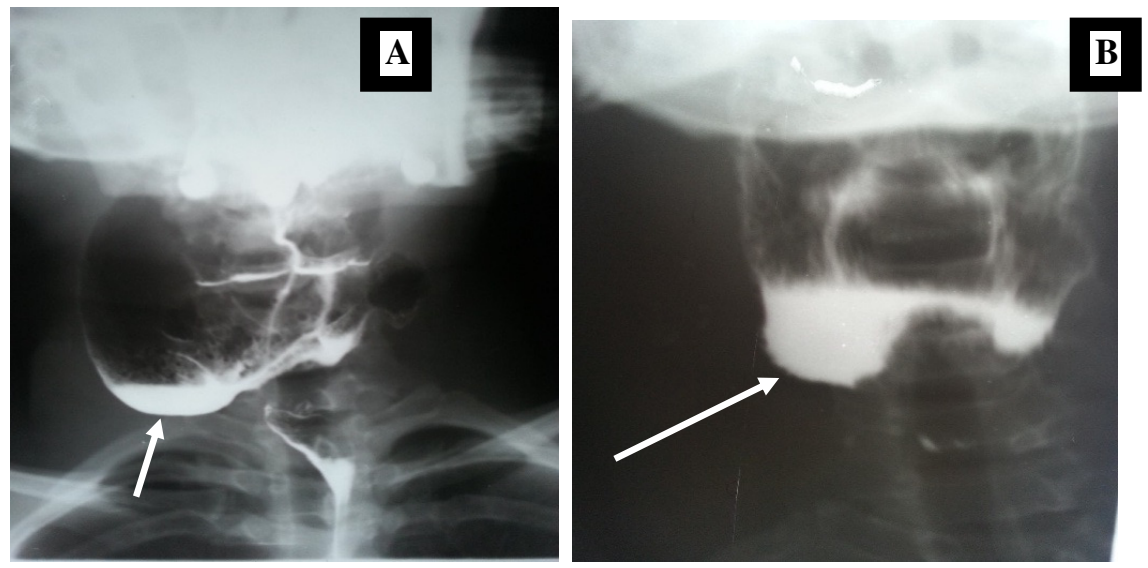

Figure 2. Barium swallow with (A) and without (B) Valsalva maneuver demonstrating the big diverticulum opening into the right Pyriform recess (Arrows)
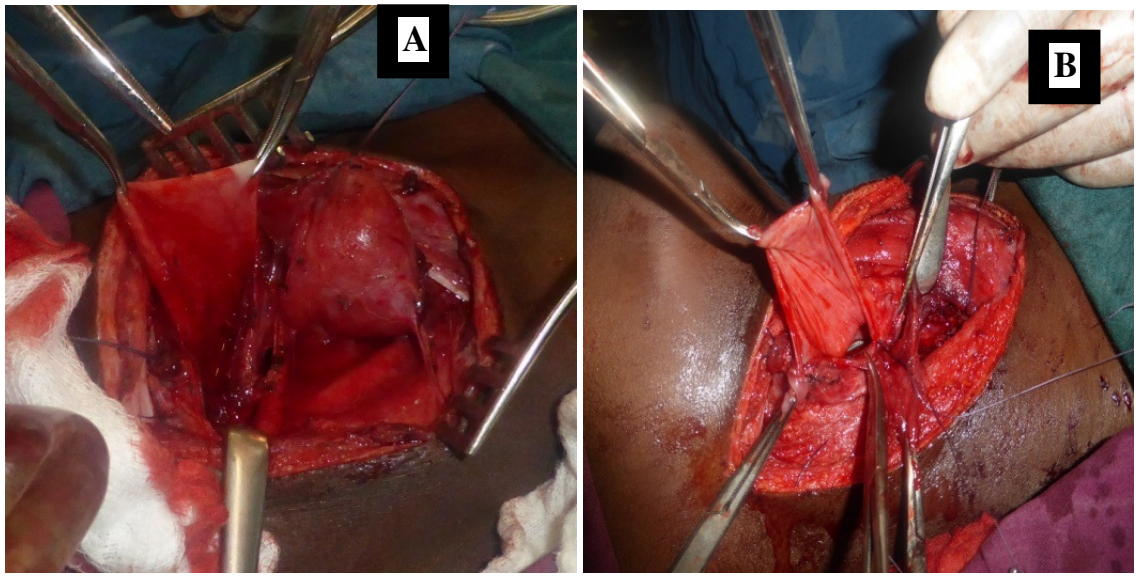

Figure 3. Diverticulum dissected (A) and opened (B)

\section{Discussion}

Many descriptions of pharyngeal wall protrusion or herniation existed since Ludlow in 1764, one would find terms like pharyngeal pouch, diverticulum or pharyngocele. ${ }^{[1]}$ Lateral pharyngeal diverticulum or Pharyngocele was first reported well in 1886 by Wheeler ${ }^{[2]}$ and since then only 55 cases were reported in the literature. ${ }^{[3]}$ These conditions are usually seen between the $6^{\text {th }}$ and $8^{\text {th }}$ decades of life due to progressive weakening of the pharyngeal wall (usually through the area between the middle and inferior constrictors and thyro-hyoid membrane) but can also be exist in younger patients. The condition occurs more commonly in males (2-3 times more common than in women) ${ }^{3}$. Lateral pharyngeal diverticulum can be either congenital or acquired and is usually suspected to occur due to the combined effects of weakened pharyngeal wall and increased intrapharyngeal pressure ${ }^{6}$. The congenital ones are thought to arise from remnants of third and fourth branchial pouches whereas acquired diverticula have been reported in individuals occupied with professions that are related with the development of increased pressure for prolonged time in the pharynx such as in Glass blowers and in individuals playing wind 


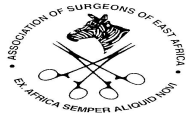

musical instruments. ${ }^{[4][6]}$ Some also suggest that prior neck surgery and trauma leading to weakened pharyngeal wall may predispose to the development of the herniation.

Our patient had history of dysphagia throughout his life which may indicate a congenital diverticulum as a result of increased pressure in the pharynx though the cause of the increased pressure that was noticed may also be from the tightly narrow cricopharynx. But if such had been the case, we would have likely seen the usual Zenker`s diverticulum in the posterior hypopharynx through the weak Killian-Jemeson dehiscence. Such is the case that it is very likely the diverticulum existed since congenitally and combined with the narrowing in the cricopharyngeal area led to its increased size which led to the lately severe dysphagia and huge swelling in the neck. The orifice of the diverticulum was wide in our patient contributing to the lack of bad odor and delayed food regurgitation commonly seen with patients having diverticula with narrow orifice.

Patients` presentation can be varied like with neck mass, dysphagia or pain, occasionally with regurgitation and dysphonia (albeit from big diverticula impinging on nerves). ${ }^{[5]}$ Investigations for lateral pharyngeal diverticula usually involve Endoscopy which would reveal the site of the diverticulum through a slit like orifice visualized around the tonsils/valeculla or pyriform sinus and in our patient due to the big diverticulum and wide orifice such visualization was not possible. Barium swallow studies also can give us better images as to the exact site and nature of the diverticula entering the pyriform recess as in our case or sometimes around the tonsils or valeculla especially when performed with Valsalva maneuver. ${ }^{7}$.

Management of such patients is usually conservative with good oral hygiene (mouth wash and gargling), avoidance of coarse particulate matter in diet like raw vegetables and whole corn especially when the diverticula are small and asymptomatic but in cases such ours when the size of the diverticulum is large

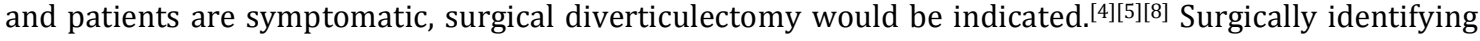
the diverticulum may be challenging at times and some surgeons would start the procedure under local anesthesia with voluntary Valsalva maneuver done by the patient to aid in localization of the pharyngocele especially when small in size and convert to general anesthesia after identifying the diverticulum ${ }^{4}$. But such endeavor was not necessary in our patient as the diverticulum was big in size and was fairly easy to identify once the dissection was carried to the level of the pharyngeal wall. Fortunately the respiratory distress in our patient that occurred post operatively was most likely from contused nerves and recovered well once the inflammation subsided over the next three days.

\section{Conclusion}

Lateral pharyngeal diverticula or pharyngocele are rare conditions and should not be mistaken for the more common Zenker`s or posterior pharyngo-esophageal diverticulum and should be included in the differential diagnosis of cystic swellings and diverticula in the pharynx when presented especially in young individuals. And as has been described in our case, barium study may be a better aid to the diagnosis in certain cases.

\section{References}

1. Norris CW. Pharyngocele of the hypopharynx. Laryngoscope 1979;89:1788-1807

2. Wheeler WI. Pharyngocele and dilatation of pharynx. Dublin J Med Sci 1886:82:349-57.

3. Gundappa D. Mahajan, James Thomas, Priya Shah, Rashmi Prashanth. Pharyngocele opening in vallecula: An unusual presentation. Medical Journal of Dr. D.Y. Patil University 2013: 6(3):318-20.

4. William G. Fowler. Lateral Pharyngeal Diverticula. Annals of Surgery January 1962; 155(1):16165.

5. P.M. Van De Ven, H.K. Schutte. The pharyngocele: infrequently encountered and easily misdiagnosed. The journal of Laryngology and Otology 1995; 109:247-49.

6. OishiKonari M, ZapaterLatorre E, MosqueraLloreda N, BasterraAlegria J. Faringocele. ActaOtorrinolaringol Esp. 2013; 64:369-370.

7. Hashem Fakhouri, Nabil Arda. Pharyngocele: A case report of a rare cause bilateral neck swelling. JRMS 2007; 14(1):53-56

8. Pinto J. A., Marquis V. B., Godoy L. B. M., Magri E. N., Brunoro M. V. F. Bilateral hypopharyngeal diverticulum. Otolaryngology 2009;141:144-145 